\title{
Reflection and Propagation of Magneto- Thermoelastic Plane Waves at Free Surfaces of a Rotating Micropolar Fibre-Reinforced Medium under G-L Theory
}

\author{
Augustine Igwebuike Anya and Aftab Khan \\ Department of Mathematics, COMSATS University Islamabad, Park Road Chak Shahzad, 44000 Islamabad, Pak- \\ istan.
}

(Received 28 October 2018; accepted 18 February 2019)

\begin{abstract}
This article is concerned with the investigation of reflection of waves at the free surface of a rotating micropolar fibre-reinforced thermoelastic medium, in the presence of a magnetic field using Green and Lindsay theory. It is observed that when a P-wave is incident on the free surface of rotating micropolar fibre-reinforced thermoelastic medium in the presence of a magnetic field, four waves are reflected; quasi-longitudinal displacement (qLD), quasi-transverse displacement (qTD), quasi-transverse micro-rotational (qTM) and quasi-thermal waves. The normal modes method, also called the harmonic solution approach, is used simultaneously with Snell's laws and Maxwell's equation, governing electromagnetic fields, in the determination of a solution for the micropolar fibrereinforced modelled problem. Amplitude ratios or reflection coefficients, which correspond to reflected waves in vertical and horizontal components, are presented analytically. Moreover, the reflection coefficients are presented using numerically simulated results in graphical form for a particular chosen material. We observe that the micropolar fibre-reinforced, rotation, and magneto-thermoelastic field parameters, have varied degree of effects on the propagation and reflection of waves in the medium. The study should be helpful in understanding the behaviour of propagation and reflection of rotating micropolar fibre-reinforced magneto-thermoelastic machination fields and on future works about behaviour of seismic waves.
\end{abstract}

\section{INTRODUCTION}

The fundamental concept of the principle of the mathematics of thermal wave starts by assuming linear proportionality of heat flux and temperature gradient as accounted by Fourier's law of heat conduction. This entails that heat transfer or exchange in materials could add value to ascertain material characteristics in continuum mechanics of fibre-reinforced composite materials. However, the analysis of stress on these fibrereinforced composite materials under the influence of magnetic field is also of interest to researchers in solid mechanics, science and technology, due to the high tensile strength and low density of fibre-reinforced materials. Magnetic fields are generally vectors giving a description of the magnetic influences of both magnetized materials and current electricity. These are felt in everyday life activities such as in permanent magnets. Hence, continuum models are capitalized on, in order to investigate the mechanical compositions of such magnetized materials. The first-grade micro-continuum, which is a material property due to deformation in terms of translational and rotational forms, plays a major role in wave propagation or reflection. It consists of microstretch, micropolar and micromorphic theories and this depends on the order in which the micro-degree is incorporated. In geophysics for instance, the reflection and refraction of seismic waves gave rise to research to investigate the earth's non exterior and macro-continuum or micro-continuum structures. Moreover, it is obvious that most large bodies such as moon, planets and earth possess angular velocity and this necessitates the study of rotational effects on reflection of magneto-thermolastic plane waves at free surfaces of micropolar fibre-reinforced medium, to which in the rotating frame of reference, the centrifugal acceleration and Coriolis effects are taken into consideration in the governing laws of motion of waves. Seismic waves are classified into body and surface waves. The former travels through the body of the medium, for example P and SV waves while the latter travels through the surface of the medium, such as Rayleigh, Love and Stoneley waves.

Furthermore, various authors have studied wave propagation in different media with effects of rotation, magnetic and thermal characteristics. Lord and Shulman, included thermal relaxation time and heat flux terms in Fourier's law of heat conduction $^{1}$ while Lindsay and Green, proceeded by adding two time relaxation thermal constants, which never violated the classical Fourier's law for a centrally symmetric body. ${ }^{2}$ Furthermore, Green and Naghdi defined three forms of relations that give wider treatment to heat flux models. ${ }^{3-5} \mathrm{Nev}-$ ertheless, various authors have also contributed to the study of micropolar elasticity theories. Eringen found the micropolar linear constitutive theories having internal friction. ${ }^{6}$ McCarthy and Eringen considered the issues in micropolar viscoelastic waves. ${ }^{7}$ Kumar, Gogna and Debnath gave account of Lamb's plane micropolar viscoelastic medium with stretch. ${ }^{8}$ Lakes and Benedict investigated noncentrosymmetry in micropolar theories of elastic media ${ }^{9}$ whereas Marin developed weak solutions in dipolar bodies with porosity and stretch. ${ }^{10,11}$ 
Biswas, Sengupta, and Debnath discussed axisymmetric wave propagation under gravity with micropolar viscolelastic halfspace. ${ }^{12}$ Kumar and Singh developed models for finding amplitude ratios as well as for reflection and refraction of micropolar elastic waves at the interface between liquid-saturated porous and micropolar elastic solids. ${ }^{13,14}$ Cowin and Nunziato discussed both linear and nonlinear theories of elastic porous media ${ }^{15,16}$ while Puri and Cowin studied the effects of voids on elastic media. ${ }^{17}$ Singh studied reflection of elastic plane surface waves with impedance boundary conditions. ${ }^{18}$ Surface waves in a fibre-reinforced media were considered by Sengupta and Nath. ${ }^{19}$ Chattopadhyay, Venkateswarlu, and Saha investigated wave reflection in fibre-reinforced medium with free and rigid boundaries. ${ }^{20}$ Chaudhary, Kaushik, and Tomar dealt with reflection and transmission of plane waves in a selfreinforced interface. ${ }^{21}$ Khan, Anya and Kaneez discussed the effects of surface waves under the influence of gravity in nonhomogeneous fibre-reinforced solid with voids. ${ }^{22}$ Tauchert investigated thermal stresses in micropolar elastic solids. ${ }^{23}$ Chattopadhyay and Choudhury obtained results on propagation, reflection and transmission of waves under magnetic influence for self-reinforced medium. ${ }^{24}$ Kumar, Sharma, and Garg worked on reflection of waves in transversely isotropic micropolar viscoelastic media with thermal effects. ${ }^{25}$ In addition, Abd-Alla, Abo-Dahab and Khan investigated the rotational behaviour of magneto-thermo-elastic surface waves in fibrereinforced viscoelastic media of higher order. ${ }^{26}$ Lata studied reflection and refraction of plane waves in a layered nonlocal elastic and anisotropic thermo-elastic medium. ${ }^{27}$ Schoenberg and Censor dealt with propagation of waves in a homogeneous rotating linear elastic medium. ${ }^{28}$ An eigenvalue approach was utilized by Sinha and Bera to solve the fundamental equations of the problems of generalized thermo-elasticity with rotation. ${ }^{29}$ Similar issues of wave propagation and reflection in the context of fibre-reinforced or thermo-elasticity with distinct parameters were studied by Deswal, Sheokand, and Kalkal, ${ }^{30}$ Roy, Acharya, and Acharya, ${ }^{31}$ Singh and Singh, ${ }^{32}$ Singh and Lianngenga, ${ }^{33}$ and Lianngenga and Singh. ${ }^{34}$

In view of the above literatures, the present investigation is concerned with propagation and reflection of magnetothermoelastic plane waves at the free surfaces of a rotating micropolar fibre-reinforced medium under G-L theory. The governing equations are solved by using natural boundary conditions. Amplitude ratios which correspond to reflected four coupled waves in both vertical and horizontal components are given analytically. Also, Mathematica software was utilized for the simulations and the results are shown graphically by considering varied parameters of the modelled system.

\section{FORMULATION OF THE PROBLEM}

The field relations for a micro-polar heat conducting fibrereinforced linearly elastic anisotropic medium with reinforcement direction ' $\vec{a}$ ' are given by: ${ }^{6,9,25}$

$$
\sigma_{i j}=S_{i j m n} E_{m n}+Q_{i j m n} \psi_{m n}-\beta_{i j}\left(1+\nu_{0} \frac{\partial}{\partial t}\right)\left(T-T_{0}\right)
$$

$m_{i j}=\hat{S}_{j i m n} \hat{E}_{m n}+Q_{m n j i} \psi_{m n}$.
The deformations and stress tensors are taken as:

$$
E_{i j}=u_{j, i}+\varepsilon_{j i m} \phi_{m}^{*} ; \quad \hat{E}_{i j}=\phi_{j, i}^{*} ; \quad \psi_{m n}=\phi_{m, n}^{*} .
$$

The balance laws in the presence of an external applied magnetic field and rotation under G-L thermo-elasticity theory are written below: $:^{2,25,26,28}$

$$
\begin{aligned}
& \sigma_{i j, i}+F_{j}=\rho\left(\ddot{u}_{j}+\Omega_{i} u_{i} \Omega_{j}-\Omega^{2} u_{j}+2 \varepsilon_{j i k} \Omega_{i} \dot{u}_{k}\right) \\
& m_{i j, i}+\varepsilon_{j m n} \sigma_{m n}=\rho J \ddot{\phi}_{j}^{*} \\
& \frac{\partial}{\partial x_{i}}\left(\kappa_{i j} \frac{\partial T}{\partial x_{j}}\right)=\rho c_{\nu}\left(\frac{\partial}{\partial t}+t_{1} \frac{\partial^{2}}{\partial t^{2}}\right) T+T_{0} \beta_{i j}\left(\frac{\partial}{\partial t}\right) E_{i j} .
\end{aligned}
$$

Let the solid medium be rotating about the $x_{3}$-axis with uniform angular velocity $\Omega$ i.e. $\vec{\Omega}=\Omega(0,0,1)$, where the thermal constant $\nu_{0}$ and $t_{1}$ in Eqs. (1) and (6) satisfy the inequalities $\nu_{0} \geq t_{1} \geq 0$. It is obvious that if $t_{1}>0$, consequently $\nu_{0}>0$, then Eq. (6) forecasts a finite speed of propagation of thermal signals and that if $\nu_{0}=t_{1}=0$, then Eqs. (1) and (6) reduce to the coupled theory of thermo-elasticity. The assumption $t_{1}=0$ and $\nu_{0}=0$ is also applicable; in this case the equation of motion continues to be affected by the temperature rate, while Eq. (6) predicts an infinite speed for the propagation of heat. In Eq. (1) we have made use of the condition $\left|T-T_{0}\right| \ll T_{0}$ to replace $T$ by $T_{0}$ in the last term of Eq. (1). Here $\kappa_{i j}$ is the conductivity tensor, $c_{\nu}$ is the specific heat at constant deformation, $\beta_{i j}$ are the thermal moduli, $\sigma_{i j}$, $m_{i j}, \phi_{j}^{*}, u_{j}$ are the stress tensor, coupled stress, micro-rotation vector and displacement vector respectively; $\rho$ is the bulk mass density, $J$ is the micro-inertia; $S_{i j m n}, \hat{S}_{j i m n}$ are characteristic constants of material and also non-symmetric properties of $S_{i j m n}, \hat{S}_{j i m n}$ and $Q_{i j m n}$ holds. For simplicity we chose $\vec{a}=\left(a_{1}, a_{2}, a_{3}\right)$ such that $\vec{a}=(1,0,0)$ is the fibre direction. $\delta_{i j}$ is the Kronecker-delta function, and $\varepsilon_{j i m}$ is the Levi-Civita tensor. An index after a comma represents a partial derivative with respect to a coordinate and a superscript dot specifies a partial derivative with respect to time. Consider the deformation in the $x_{1} x_{2}$-plane and $\phi^{*}=\left(0,0, \phi_{3}^{*}\right) . F_{i}=\mu_{0} \varepsilon_{i j k} J_{j} H_{k}$. We consider the linearized Maxwell equations governing the electromagnetic field for a perfectly conducting medium as: $\varepsilon_{i j k} H_{k, j}=\varepsilon_{0} \varepsilon_{i j k} J_{j} \dot{E}_{k}, \varepsilon_{i j k} E_{k, j}=-\mu_{0} \dot{H}_{i}, H_{i, i}=0$, $E_{i, i}=0, E_{i}=\mu_{0} \varepsilon_{i j k} \dot{u}_{j} H_{k}$, where $H_{i}=H_{0} \delta_{i 3}+h_{i}$, $h_{i}$ is the induced magnetic field and $\varepsilon_{0}$ is the electric permeability and the material lies in the $x_{1} x_{2}$-plane. Then the magnetic force is as follows: $F_{i}=\mu_{0} H_{0}^{2}\left(e_{, i}-\varepsilon_{0} \mu_{0} \ddot{u}_{i}\right)$, $F_{3}=0$ and $h_{i}\left(x_{1}, x_{2}, x_{3}\right)=-u_{k, k} \delta_{i 3}$, where $e=u_{1,1}+u_{2,2}$, $i=j=1,2,3$.

In these equations, $F_{i}$ indicates magnetic force, $J_{i}$ is the current density, $H_{i}$ is the magnetic vector field and $\mu_{0}$ is the magnetic permeability. Considering the fact that tensors are not symmetric in micropolar media, and without rotation, Eqs. (4)-(6) in component form can be written as follows:

$$
\begin{aligned}
B_{1} u_{1,11}+ & \left(B_{2}+B_{3}\right) u_{2,12}+B_{4} u_{1,22}+B_{1}^{*} \phi_{3,2}^{*}- \\
& \beta_{1}\left(1+\nu_{0} \frac{\partial}{\partial t}\right) T_{, 1}=\left(\rho+\varepsilon_{0} \mu_{0}^{2} H_{0}^{2}\right) \ddot{u}_{1} ; \\
B_{5} u_{2,11}+ & B_{2} u_{1,12}+B_{6} u_{2,22}-B_{5} \phi_{3,1}^{*}- \\
& \beta_{1}\left(1+\nu_{0} \frac{\partial}{\partial t}\right) T_{, 2}=\left(\rho+\varepsilon_{0} \mu_{0}^{2} H_{0}^{2}\right) \ddot{u}_{2} ;
\end{aligned}
$$




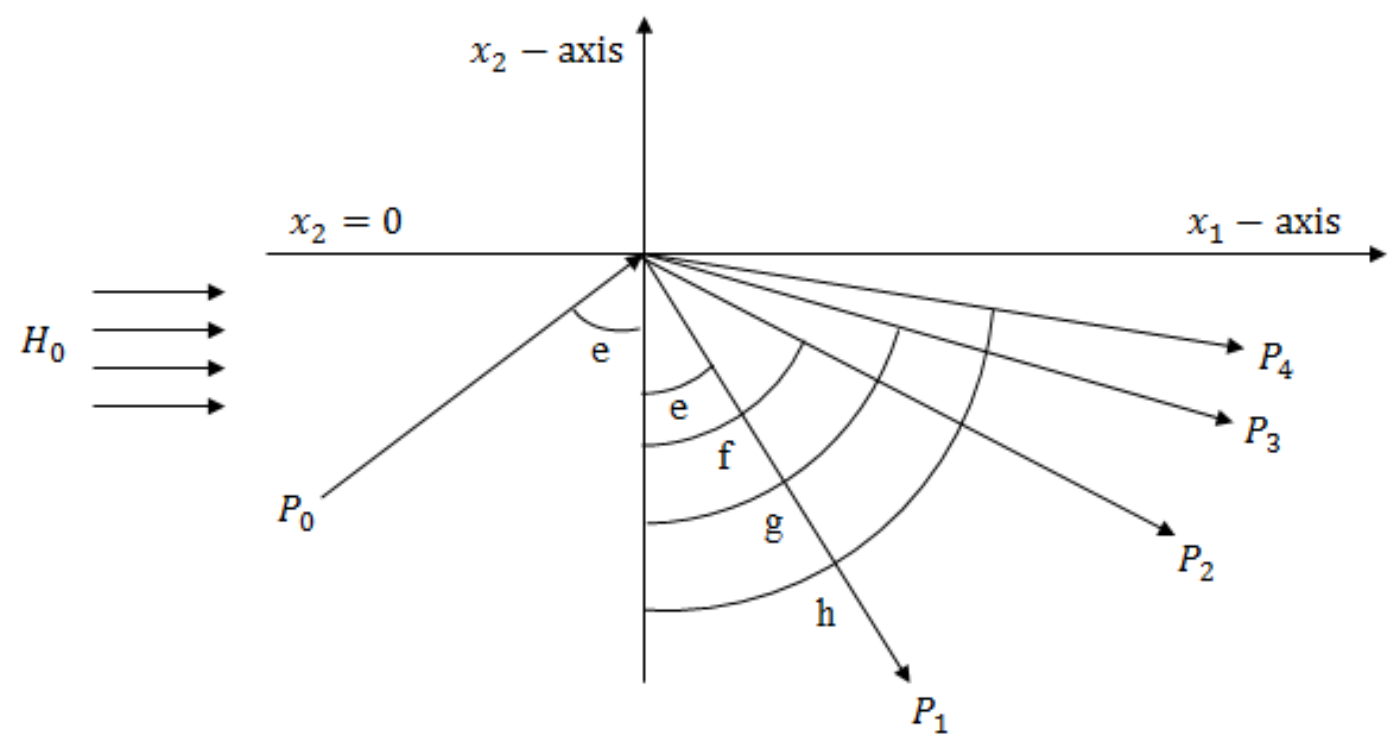

Figure 1. Schematic of the problem showing the incident and reflected waves.

$$
\begin{aligned}
& B_{5} \phi_{3,11}^{*}+B_{4} \phi_{3,22}^{*}-2 B_{4} \phi_{3}^{*}+B_{4}\left(u_{2,1}-u_{1,2}\right)=\rho J \ddot{\phi}_{3}^{*} ; \\
& K_{1} T_{, i i}=\rho c_{\nu}\left(\dot{T}+\tau_{0} \ddot{T}\right)+T_{0} \beta_{1} \dot{u}_{i, i}
\end{aligned}
$$

where

$$
\begin{aligned}
& B_{1}=\lambda+\beta+2 \alpha-2 \mu_{T}+4 \mu_{L}+\mu_{0} H_{0}^{2} ; \\
& B_{2}=\lambda+\alpha+\mu_{0} H_{0}^{2} ; \quad B_{3}=2\left(\mu_{L}-\mu_{T}\right) ; \quad B_{4}=2 \mu_{T} \\
& B_{5}=2 \mu_{L} ; \quad B_{6}=\lambda+2 \mu_{T}+\mu_{0} H_{0}^{2} ; \quad B_{1}^{*}=B_{4}-B_{3} .
\end{aligned}
$$

\section{NORMAL MODES ANALYSIS AND SOLUTION OF THE PROBLEM}

Consider a homogeneous rotating micropolar fibrereinforced thermo-elastic anisotropic medium under the influenced of magnetic fields occupying the half-space $x_{2} \leq 0$ and plane waves incident at the free boundary $x_{2}=0$ at an angle $\theta$ with the $x_{2}$-axis. Let normal mode analysis be applicable such that the incident waves have the displacement chosen as:

$$
\begin{aligned}
& u_{1}=R e^{i\left\{k\left(x_{1} \sin \theta+x_{2} \cos \theta\right)-\omega t\right\}} \\
& u_{2}=P e^{i\left\{k\left(x_{1} \sin \theta+x_{2} \cos \theta\right)-\omega t\right\}} \\
& \phi_{3}^{*}=\phi_{0}^{*} e^{i\left\{k\left(x_{1} \sin \theta+x_{2} \cos \theta\right)-\omega t\right\}} \\
& \left(T-T_{0}\right)=\theta=\theta_{0} e^{i\left\{k\left(x_{1} \sin \theta+x_{2} \cos \theta\right)-\omega t\right\}}
\end{aligned}
$$

where $R, P, \phi_{0}^{*}$, and $\theta_{0}$ are amplitudes of $u_{1}, u_{2}, \phi_{3}^{*}$, and $\theta$ respectively. $\omega$ is the angular velocity or frequency, $c=\frac{\omega}{k}$ is the phase velocity of the wave, and $k$ is the wave number. We make use of Eqs. (12)-(15) into Eqs. (7)-(10) respectively, and with rotation of the media, yields:

$$
\begin{gathered}
{\left[D_{1}-\left\{\frac{\Omega^{2}}{k^{2}} \rho+c^{2}\left(\varepsilon_{0} \mu_{0}^{2} H_{0}^{2}+\rho\right)\right\}\right] R+} \\
\left\{\left(B_{2}+B_{3}\right) \cos \theta \sin \theta+2 i c \frac{\Omega}{k} \rho\right\} P+ \\
\left\{i \frac{1}{k} \beta_{1}\left(1-i \omega \nu_{0}\right) \sin \theta\right\} \theta_{0}=0 \\
\left\{B_{2} \cos \theta \sin \theta-2 i c \frac{\Omega}{k} \rho\right\} R+ \\
\quad\left[D_{2}-\left\{\frac{\Omega^{2}}{k^{2}} \rho+c^{2}\left(\varepsilon_{0} \mu_{0}^{2} H_{0}^{2}+\rho\right)\right\}\right] P+ \\
\left\{i \frac{1}{k} \beta_{1}\left(1-i \omega \nu_{0}\right) \cos \theta\right\} \theta_{0}=0 ; \\
\left\{i k B_{4} \cos \theta\right\} R-\left\{i k B_{4} \sin \theta\right\} P+ \\
\left\{k^{2} D_{3}+2 B_{4}-\rho J k^{2} c^{2}\right\} \phi_{0}^{*}=0 \\
-\left(\beta_{1} k T_{0} \omega \sin \theta\right) R-\left(\beta_{1} k T_{0} \omega \cos \theta\right) P+ \\
\left\{\rho c_{\nu}\left(i \omega+\tau_{0} k^{2} c^{2}\right)-K_{1} k^{2}\right\} \theta_{0}=0
\end{gathered}
$$

where $D_{1}=B_{1} \sin ^{2} \theta+B_{4} \cos ^{2} \theta, D_{2}=B_{5} \sin ^{2} \theta+$ $B_{6} \cos ^{2} \theta, D_{3}=B_{5} \sin ^{2} \theta+B_{4} \cos ^{2} \theta$. For a non-trivial solution, Eqs. (17)-(19), becomes:

$$
d^{4}+C_{1} d^{3}+C_{2} d^{2}+C_{3} d+C_{4}=0
$$

where $d=c^{2}$, this means that the characteristic Eq. (20) with complex coefficients $C_{1}, C_{2}, C_{3}$, and $C_{4}$ (See appendix) yields four complex roots; which shows that four waves propagate, with complex phase velocities: $c_{1}, c_{2}, c_{3}$, and $c_{4}$ corresponding to wave numbers $k_{1}, k_{2}, k_{3}$, and $k_{4}$, respectively. So this stipulates also that the two dimensional model of the magnetothermo-elastic rotating micropolar fibre-reinforced half space medium under G-L theory has four reflected waves, a quasi$\mathrm{P}$ wave, a quasi-SV wave, a quasi-transverse micro-rotational 
wave and a thermal wave travelling in the medium, if we assume any one of the four waves is incident at the free surface.

\section{INCIDENT QUASI-P AND REFLECTED WAVES AT THE FREE BOUNDARY}

In Fig. 1, when a quasi-P wave $\left(P_{0}\right)$ is incident at the boundary $x_{2}=0$ of the rotating micropolar fibre-reinforced anisotropic semi-infinite medium under magneto-thermo effects, then there exist reflected waves as quasi-P $\left(P_{1}\right)$, quasi$\mathrm{SV}\left(P_{2}\right)$, quasi-TM $\left(P_{3}\right)$ and quasi-thermal waves $\left(P_{4}\right)$-see Fig. 1.

We assume the total displacements in the following form:

$$
\left\{u_{1}, u_{2}, \phi_{3}^{*}, \theta\right\}=\sum_{l=0}^{4}\left\{R_{l}, P_{l}, \phi_{0}^{*}, \theta_{0}\right\} e^{\left(i S_{l}\right)}
$$

where $S_{0}=k_{0}\left[x_{1} \sin (e)+x_{2} \cos (e)-c_{1} t\right] ; \quad S_{1}=$ $k_{1}\left[x_{1} \sin (e)-x_{2} \cos (e)-c_{1} t\right] ; \quad S_{2}=k_{2}\left[x_{1} \sin (f)-\right.$ $\left.x_{2} \cos (f)-c_{2} t\right] ; S_{3}=k_{3}\left[x_{1} \sin (g)-x_{2} \cos (g)-c_{3} t\right]$; $S_{4}=k_{4}\left[x_{1} \sin (h)-x_{2} \cos (h)-c_{4} t\right]$.

The incident and reflected waves given by Eq. (21) must satisfy Eqs. (7)-(8) of motion as a result of consistency conditions; thus Eq. (7) becomes: $:^{31,32}$

$$
\begin{aligned}
& \left\{D_{1}(e)-l_{1}\right\} R_{0}+\left\{\left(A_{2}+A_{3}\right) \sin (e) \cos (e)+2 i c_{1} \frac{\Omega}{k_{1}} \rho\right\} P_{0}- \\
& i\left\{\frac{i}{k_{0}} A_{1}^{*} \cos (e)\right\} \phi_{0}^{*}+\left\{\frac{i}{k_{0}} \xi \sin (e)\right\} \theta_{0}=0 ; \\
& \left\{D_{1}(e)-l_{1}\right\} R_{1}-\left\{\left(A_{2}+A_{3}\right) \sin (e) \cos (e)-2 i c_{1} \frac{\Omega}{k_{1}} \rho\right\} P_{1}- \\
& \quad i\left\{\frac{i}{k_{1}} A_{1}^{*} \cos (e)\right\} \phi_{0}^{*}+\left\{\frac{i}{k_{1}} \xi \sin (e)\right\} \theta_{0}=0 ; \\
& \left\{D_{1}(f)-l_{3}\right\} R_{2}-\left\{\left(A_{2}+A_{3}\right) \sin (f) \cos (f)-2 i c_{1} \frac{\Omega}{k_{2}} \rho\right\} P_{2}- \\
& \quad i\left\{\frac{i}{k_{2}} A_{1}^{*} \cos (f)\right\} \phi_{0}^{*}+\left\{\frac{i}{k_{2}} \xi \sin (f)\right\} \theta_{0}=0 ; \\
& \left\{D_{1}(g)-l_{3}\right\} R_{3}-\left\{\left(A_{2}+A_{3}\right) \sin (g) \cos (g)-2 i c_{1} \frac{\Omega}{k_{3}} \rho\right\} P_{3}- \\
& i\left\{\frac{i}{k_{3}} A_{1}^{*} \cos (g)\right\} \phi_{0}^{*}+\left\{\frac{i}{k_{3}} \xi \sin (g)\right\} \theta_{0}=0 ; \\
& \left\{D_{1}(h)-l_{4}\right\} R_{4}-\left\{\left(A_{2}+A_{3}\right) \sin (h) \cos (h)-2 i c_{1} \frac{\Omega}{k_{4}} \rho\right\} P_{4}- \\
& i\left\{\frac{i}{k_{4}} A_{1}^{*} \cos (h)\right\} \phi_{0}^{*}+\left\{\frac{i}{k_{4}} \xi \sin (h)\right\} \theta_{0}=0 .
\end{aligned}
$$

Equation (22) is derived by the application of Snell's law such that: $c_{1} k_{0}=c_{1} k_{1}=c_{2} k_{2}=c_{3} k_{3}=c_{4} k_{4}$ and also $k_{0} \sin (e)=k_{1} \sin (e)=k_{2} \sin (f)=k_{3} \sin (g)=k_{4} \sin (h)$. Note that $k_{0}=k_{1}$, where the wave speeds are made of material parameters such that in Eq (22), $A=B$. And thus, Eq. (22) is rewritten as:

$$
\begin{aligned}
& R_{0}=-s_{1} P_{0}+s_{2} \phi_{0}^{*}-s_{3} \theta_{0} \\
& R_{1}=s_{1} P_{1}+s_{4} \phi_{0}^{*}-s_{5} \theta_{0} \\
& R_{2}=s_{6} P_{2}+s_{7} \phi_{0}^{*}-s_{8} \theta_{0} \\
& R_{3}=s_{9} P_{3}+s_{10} \phi_{0}^{*}-s_{11} \theta_{0} ; \\
& R_{4}=s_{12} P_{4}+s_{13} \phi_{0}^{*}-s_{14} \theta_{0} ;
\end{aligned}
$$

where:

$$
\begin{aligned}
s_{1} & =\frac{\left(B_{2}+B_{3}\right) \sin (e) \cos (e)+2 i c_{1} \frac{\Omega}{k_{1}} \rho}{D_{1}(e)-l_{1}} ; \\
s_{2} & =\frac{i B_{1}^{*} \cos (e)}{k_{0}\left\{D_{1}(e)-l_{1}\right\}} ; \quad s_{3}=\frac{i \xi \sin (e)}{k_{0}\left\{D_{1}(e)-l_{1}\right\}} ; \\
s_{4} & =\frac{i \xi B_{1}^{*} \cos (e)}{k_{1}\left\{D_{1}(e)-l_{1}\right\}} ; \quad s_{5}=\frac{i \xi \sin (e)}{k_{1}\left\{D_{1}(e)-l_{1}\right\}} ; \\
s_{6} & =\frac{\left(B_{2}+B_{3}\right) \sin (f) \cos (f)-2 i c_{2} \frac{\Omega}{k_{2}} \rho}{D_{1}(f)-l_{2}} ; \\
s_{7} & =\frac{i B_{1}^{*} \cos (f)}{k_{2}\left\{D_{1}(f)-l_{2}\right\}} ; \quad s_{8}=\frac{i \xi \sin (f)}{k_{2}\left\{D_{1}(f)-l_{2}\right\}} ; \\
s_{9} & =\frac{\left(B_{2}+B_{3}\right) \sin (g) \cos (g)-2 i c_{3} \frac{\Omega}{k_{3}} \rho}{D_{1}(g)-l_{3}} ; \\
s_{10} & =\frac{i B_{1}^{*} \cos (g)}{k_{3}\left\{D_{1}(g)-l_{3}\right\}} ; \quad s_{11}=\frac{i \xi \sin (g)}{k_{3}\left\{D_{1}(g)-l_{3}\right\}} ; \\
s_{12} & =\frac{\left(B_{2}+B_{3}\right) \sin (h) \cos (h)-2 i c_{4} \frac{\Omega}{k_{4}} \rho}{D_{1}(h)-l_{4}} ; \\
s_{13} & =\frac{i B_{1}^{*} \cos (h)}{k_{4}\left\{D_{1}(h)-l_{4}\right\}} ; \quad s_{14}=\frac{i \xi \sin (h)}{k_{4}\left\{D_{1}(h)-l_{4}\right\}} ; \\
\xi & =\beta_{1}\left(1-i \omega \nu_{0}\right) ; \\
l_{j} & =\left(\frac{\Omega^{2}}{k_{j}^{2}} \rho+c_{j}^{2}\left(\varepsilon_{0} \mu_{0}^{2} H_{0}^{2}+\rho\right)\right), \quad j=1,2,3,4 .
\end{aligned}
$$

\subsection{Free Boundary Conditions}

$\sigma_{i 2}+\bar{\sigma}_{i 2}=0, i=1,2, m_{23}=0$, and $T_{, 2}=0$ at $x_{2}=0$. Maxwell's stresses are given as: ${ }^{26} \bar{\sigma}_{i j}=\mu_{0} H_{0}\left[H_{i} h_{j}+\right.$ $\left.H_{j} h_{i}-H_{k} h_{k} \delta_{i j}\right] \Rightarrow \bar{\sigma}_{i j}=\mu_{0} H_{0}\left[\begin{array}{ccc}-h_{3} & 0 & h_{1} \\ 0 & -h_{3} & h_{2} \\ h_{1} & h_{2} & h_{3}\end{array}\right]$; and $h_{3}=-H_{0}\left(u_{1,1}+u_{2,2}\right) ; h_{1}=h_{2}=0$.

For $i=1, \sigma_{12}+\bar{\sigma}_{12}=0$, but $\bar{\sigma}_{12}=0 \Rightarrow \sigma_{12}=0$; for $i=2, \sigma_{22}+\bar{\sigma}_{22}=0 \Rightarrow \sigma_{22}+\mu_{0} H_{0}^{2}\left(u_{1,1}+u_{2,2}\right)=0$;

$m_{23}=0 \Rightarrow \phi_{3,2}^{*}=0$

and $T_{, 2}=0$.

Thus, from Eq. (23), we have:

$$
\begin{aligned}
& \frac{R_{1}}{R_{0}}=\frac{s_{1} \frac{P_{1}}{P_{0}}+s_{4} \frac{\theta_{0}^{*}}{P_{0}}-s_{5} \frac{\theta_{0}}{P_{0}}}{-s_{3} \frac{\theta_{0}}{P_{0}}+s_{2} \frac{\theta_{0}^{*}}{P_{0}}-s_{1}} \\
& \frac{R_{2}}{R_{0}}=\frac{s_{6} \frac{P_{2}}{P_{0}}+s_{7} \frac{\theta_{0}^{*}}{P_{0}}-s_{8} \frac{\theta_{0}}{P_{0}}}{-s_{3} \frac{\theta_{0}}{P_{0}}+s_{2} \frac{\theta_{0}^{*}}{P_{0}}-s_{1}} \\
& \frac{R_{3}}{R_{0}}=\frac{s_{9} \frac{P_{3}}{P_{0}}+s_{10} \frac{\theta_{0}^{*}}{P_{0}}-s_{11} \frac{\theta_{0}}{P_{0}}}{-s_{3} \frac{\theta_{0}}{P_{0}}+s_{2} \frac{\theta_{0}^{*}}{P_{0}}-s_{1}} \\
& \frac{R_{4}}{R_{0}}=\frac{s_{12} \frac{P_{4}}{P_{0}}+s_{13} \frac{\theta_{0}^{*}}{P_{0}}-s_{14} \frac{\theta_{0}}{P_{0}}}{-s_{3} \frac{\theta_{0}}{P_{0}}+s_{2} \frac{\theta_{0}^{*}}{P_{0}}-s_{1}}
\end{aligned}
$$


and also by using Eqs. (25)-(28), we have:

$$
\begin{aligned}
& a_{11} \frac{P_{1}}{P_{0}}+a_{12} \frac{P_{2}}{P_{0}}+a_{13} \frac{P_{3}}{P_{0}}+a_{14} \frac{P_{4}}{P_{0}}=a_{15} ; \\
& a_{21} \frac{P_{1}}{P_{0}}+a_{22} \frac{P_{2}}{P_{0}}-a_{23} \frac{P_{3}}{P_{0}}+a_{24} \frac{P_{4}}{P_{0}}=-a_{25} ; \\
& a_{31} \frac{P_{1}}{P_{0}}-a_{32} \frac{P_{2}}{P_{0}}+a_{33} \frac{P_{3}}{P_{0}}+a_{34} \frac{P_{4}}{P_{0}}=-a_{35} ; \\
& a_{41} \frac{P_{1}}{P_{0}}+a_{42} \frac{P_{2}}{P_{0}}+a_{43} \frac{P_{3}}{P_{0}}-a_{44} \frac{P_{4}}{P_{0}}=a_{45} ;
\end{aligned}
$$

where:

$$
\begin{aligned}
& a_{11}=k_{1} \sin (e) ; \quad a_{12}=k_{2} \sin (f) ; \quad a_{13}=k_{3} \sin (g) ; \\
& a_{14}=k_{4} \sin (h) ; \quad a_{15}=-\left\{k_{0} \sin (e)+\frac{i \phi_{0}^{*}}{P_{0}}\right\} ; \\
& a_{21}=\left\{L_{1} s_{1} \sin (e)-L_{2} \cos (e)\right\} k_{1} ; \\
& a_{22}=\left\{L_{1} s_{6} \sin (f)-L_{2} \cos (f)\right\} k_{2} ; \\
& a_{23}=-\left\{L_{1} s_{9} \sin (g)-L_{2} \cos (g)\right\} k_{3} ; \\
& a_{24}=\left\{L_{1} s_{12} \sin (h)-L_{2} \cos (h)\right\} k_{4} ; \\
& a_{25}=-\left\{L_{1} s_{1} \sin (e)-L_{2} \cos (e)\right\} k_{0}- \\
& L_{1}\left\{\left(s_{2} \frac{\phi_{0}^{*}}{P_{0}}+s_{3} \frac{\theta_{0}}{P_{0}}\right) k_{0} \sin (e)+\left(s_{4} \frac{\phi_{0}^{*}}{P_{0}}+s_{5} \frac{\theta_{0}}{P_{0}}\right) k_{1} \sin (e)+\right. \\
& \left(s_{7} \frac{\phi_{0}^{*}}{P_{0}}+s_{8} \frac{\theta_{0}}{P_{0}}\right) k_{2} \sin (f)+\left(s_{10} \frac{\phi_{0}^{*}}{P_{0}}+s_{11} \frac{\theta_{0}}{P_{0}}\right) k_{3} \sin (g)+ \\
& \left.\left(s_{13} \frac{\phi_{0}^{*}}{P_{0}}+s_{14} \frac{\theta_{0}}{P_{0}}\right) k_{4} \sin (h)\right\}+i \xi \frac{\theta_{0}}{P_{0}} ;
\end{aligned}
$$$$
a_{31}=-B_{1} s_{1} a_{11}^{2}+\left(B_{2}+B_{3}\right) a_{11} k_{1} \cos (e)+2 i \rho c_{1} k_{1} \Omega-
$$$$
B_{4} s_{1} k_{1}^{2} \cos ^{2}(e)+\left(\Omega^{2} \rho+c_{1}^{2} k_{1}^{2}\left(\varepsilon_{0} \mu_{0}^{2} H_{0}^{2}+\rho\right)\right) s_{1} \text {; }
$$$$
a_{32}=-\left\{-B_{1} s_{6} a_{12}^{2}+\left(B_{2}+B_{3}\right) a_{12} k_{2} \cos (f)+2 i \rho c_{2} k_{2} \Omega-\right.
$$$$
\left.B_{4} s_{6} k_{2}^{2} \cos ^{2}(f)+\left(\Omega^{2} \rho+c_{2}^{2} k_{2}^{2}\left(\varepsilon_{0} \mu_{0}^{2} H_{0}^{2}+\rho\right)\right) s_{6}\right\} ;
$$$$
a_{33}=-B_{1} s_{9} a_{13}^{2}+\left(B_{2}+B_{3}\right) a_{13} k_{3} \cos (g)+2 i \rho c_{3} k_{3} \Omega-
$$$$
B_{4} s_{9} k_{3}^{2} \cos ^{2}(g)+\left(\Omega^{2} \rho+c_{3}^{2} k_{3}^{2}\left(\varepsilon_{0} \mu_{0}^{2} H_{0}^{2}+\rho\right)\right) s_{9} ;
$$$$
a_{34}=-B_{1} s_{12} a_{14}^{2}+\left(B_{2}+B_{3}\right) a_{14} k_{4} \cos (h)+2 i \rho c_{4} k_{4} \Omega-
$$$$
B_{4} s_{12} k_{4}^{2} \cos ^{2}(h)+\left(\Omega^{2} \rho+c_{4}^{2} k_{4}^{2}\left(\varepsilon_{0} \mu_{0}^{2} H_{0}^{2}+\rho\right)\right) s_{12} \text {; }
$$$$
a_{35}=-\left\{B _ { 1 } \left\{\left(-s_{1}+s_{2} \frac{\phi_{0}^{*}}{P_{0}}+s_{3} \frac{\theta_{0}}{P_{0}}\right) k_{0}^{2} \sin ^{2}(e)+\right.\right.
$$$$
\left(s_{4} \frac{\phi_{0}^{*}}{P_{0}}+s_{5} \frac{\theta_{0}}{P_{0}}\right) a_{11}^{2}+\left(s_{7} \frac{\phi_{0}^{*}}{P_{0}}+s_{8} \frac{\theta_{0}}{P_{0}}\right) a_{12}^{2}+
$$$$
\left.\left(s_{10} \frac{\phi_{0}^{*}}{P_{0}}+s_{11} \frac{\theta_{0}}{P_{0}}\right) a_{13}^{2}+\left(s_{13} \frac{\phi_{0}^{*}}{P_{0}}+s_{14} \frac{\theta_{0}}{P_{0}}\right) a_{14}^{2}\right\}+
$$

$\left(B_{2}+B_{3}\right) k_{0}^{2} \cos (e) \cos (e)+2 i \rho c_{1} \Omega+$

$$
B_{4}\left\{\left(-s_{1}+s_{2} \frac{\phi_{0}^{*}}{P_{0}}+s_{3} \frac{\theta_{0}}{P_{0}}\right) k_{0}^{2} \cos ^{2}(e)+\right.
$$$$
\left(s_{4} \frac{\phi_{0}^{*}}{P_{0}}+s_{5} \frac{\theta_{0}}{P_{0}}\right) k_{1}^{2} \cos ^{2}(e)+\left(s_{7} \frac{\phi_{0}^{*}}{P_{0}}+s_{8} \frac{\theta_{0}}{P_{0}}\right) k_{2}^{2} \cos ^{2}(f)+
$$$$
\left(s_{10} \frac{\phi_{0}^{*}}{P_{0}}+s_{11} \frac{\theta_{0}}{P_{0}}\right) k_{3}^{2} \cos ^{2}(g)+
$$$$
\left.\left(s_{13} \frac{\phi_{0}^{*}}{P_{0}}+s_{14} \frac{\theta_{0}}{P_{0}}\right) k_{4}^{2} \cos ^{2}(h)\right\}-
$$$$
\left(-s_{1}+s_{2} \frac{\phi_{0}^{*}}{P_{0}}+s_{3} \frac{\theta_{0}}{P_{0}}\right)\left(\Omega^{2} \rho+c_{1}^{2} k_{0}^{2}\left(\varepsilon_{0} \mu_{0}^{2} H_{0}^{2}+\rho\right)\right)-
$$

$$
\begin{aligned}
& \left(s_{4} \frac{\phi_{0}^{*}}{P_{0}}+s_{5} \frac{\theta_{0}}{P_{0}}\right)\left(\Omega^{2} \rho+c_{1}^{2} k_{1}^{2}\left(\varepsilon_{0} \mu_{0}^{2} H_{0}^{2}+\rho\right)\right)- \\
& \left(s_{7} \frac{\phi_{0}^{*}}{P_{0}}+s_{8} \frac{\theta_{0}}{P_{0}}\right)\left(\Omega^{2} \rho+c_{2}^{2} k_{2}^{2}\left(\varepsilon_{0} \mu_{0}^{2} H_{0}^{2}+\rho\right)\right)- \\
& \left(s_{10} \frac{\phi_{0}^{*}}{P_{0}}+s_{11} \frac{\theta_{0}}{P_{0}}\right)\left(\Omega^{2} \rho+c_{3}^{2} k_{3}^{2}\left(\varepsilon_{0} \mu_{0}^{2} H_{0}^{2}+\rho\right)\right)- \\
& \left(s_{13} \frac{\phi_{0}^{*}}{P_{0}}+s_{14} \frac{\theta_{0}}{P_{0}}\right)\left(\Omega^{2} \rho+c_{4}^{2} k_{4}^{2}\left(\varepsilon_{0} \mu_{0}^{2} H_{0}^{2}+\rho\right)\right)-
\end{aligned}
$$

$\left.i \xi \frac{\theta_{0}}{P_{0}}\left\{k_{0} \sin (e)+a_{11}+a_{12}+a_{13}+a_{14}\right\}\right\} ;$

$a_{41}=-\left(B_{5}+B_{6}\right) a_{11}^{2}+\left(B_{2} a_{11} k_{1} \cos (e)-2 i \rho c_{1} k_{1} \Omega\right) s_{1}+$ $\Omega^{2} \rho+c_{1}^{2} k_{1}^{2}\left(\varepsilon_{0} \mu_{0}^{2} H_{0}^{2}+\rho\right) ;$

$a_{42}=-\left(B_{5}+B_{6}\right) a_{12}^{2}+\left(B_{2} a_{12} k_{2} \cos (f)-2 i \rho c_{2} k_{2} \Omega\right) s_{6}+$ $\Omega^{2} \rho+c_{2}^{2} k_{2}^{2}\left(\varepsilon_{0} \mu_{0}^{2} H_{0}^{2}+\rho\right) ;$

$a_{43}=-\left(B_{5}+B_{6}\right) a_{13}^{2}+\left(B_{2} a_{13} k_{3} \cos (g)-2 i \rho c_{3} k_{3} \Omega\right) s_{9}+$ $\Omega^{2} \rho+c_{3}^{2} k_{3}^{2}\left(\varepsilon_{0} \mu_{0}^{2} H_{0}^{2}+\rho\right) ;$

$a_{44}=-\left\{-\left(B_{5}+B_{6}\right) a_{14}^{2}+\left(B_{2} a_{14} k_{4} \cos (h)-\right.\right.$

$\left.\left.2 i \rho c_{4} k_{4} \Omega\right) s_{12}+\Omega^{2} \rho+c_{4}^{2} k_{4}^{2}\left(\varepsilon_{0} \mu_{0}^{2} H_{0}^{2}+\rho\right)\right\}$;

$a_{45}=\left(B_{5} \sin ^{2}(e)+B_{6} \cos ^{2}(e)\right) k_{0}^{2}-$

$\left(\Omega^{2} \rho+c_{1}^{2} k_{0}^{2}\left(\varepsilon_{0} \mu_{0}^{2} H_{0}^{2}+\rho\right)\right)+$

$\left(s_{2} \frac{\phi_{0}^{*}}{P_{0}}+s_{3} \frac{\theta_{0}}{P_{0}}\right)\left(B_{2} k_{0}^{2} \cos (e) \sin (e)-2 i \rho c_{1} k_{1} \Omega\right)-$

$\left(s_{4} \frac{\phi_{0}^{*}}{P_{0}}+s_{5} \frac{\theta_{0}}{P_{0}}\right)\left(k_{1} B_{2} a_{11} \cos (e)-2 i \rho c_{1} k_{1} \Omega\right)-$

$\left(s_{7} \frac{\phi_{0}^{*}}{P_{0}}+s_{8} \frac{\theta_{0}}{P_{0}}\right)\left(k_{2} B_{2} a_{12} \cos (f)-2 i \rho c_{2} k_{2} \Omega\right)-$

$\left(s_{10} \frac{\phi_{0}^{*}}{P_{0}}+s_{11} \frac{\theta_{0}}{P_{0}}\right)\left(k_{3} B_{2} a_{13} \cos (g)-2 i \rho c_{3} k_{3} \Omega\right)-$

$\left(s_{13} \frac{\phi_{0}^{*}}{P_{0}}+s_{14} \frac{\theta_{0}}{P_{0}}\right)\left(k_{4} B_{2} a_{15} \cos (h)-2 i \rho c_{4} k_{4} \Omega\right)+$

$i B_{5} \frac{\phi_{0}^{*}}{P_{0}}\left\{k_{0} \sin (e)+a_{11}+a_{12}+a_{13}+a_{14}\right\} ;$

$L_{1}=B_{2} ; \quad$ and $\quad L_{2}=B_{6}$.

Equation (30) can be solved for $Z_{i}^{*}=\left|\frac{P_{i}}{P_{0}}\right|, i=1,2,3$, and 4 , simultaneously as the amplitude ratios or reflection coefficients along vertical direction and hence amplitude ratios $Z_{i}=\left|\frac{R_{i}}{R_{0}}\right|, i=1,2,3$, and 4 , along horizontal direction can be obtained from Eq. (29).

\section{COMPUTATIONAL RESULTS AND DISCUSSION}

To study the effects of magnetic field, and thermal parameters of reflection coefficients of plane waves in a rotating micropolar fibre-reinforced material under Greens and Lindsay theory of thermos-elasticity, the numerical fibre-reinforced physical constants ${ }^{30}$ and other parameters are taken as: $\lambda=$ $5.65 \times 10^{10} \mathrm{kgm}^{-1} \mathrm{~s}^{-2} ; \mu_{T}=2.46 \times 10^{10} \mathrm{kgm}^{-1} \mathrm{~s}^{-2}$; $\mu_{L}=5.66 \times 10^{10} \mathrm{kgm}^{-1} \mathrm{~s}^{-2} ; \rho=2660 \mathrm{kgm}^{-3} ; \alpha=$ $-1.28 \times 10^{9} \mathrm{kgm}^{-1} \mathrm{~s}^{-2} ; \beta=220.90 \times 10^{9} \mathrm{kgm}^{-1} \mathrm{~s}^{-2}$; $H_{0}=100 ; k_{0}=1 ; \Omega=10 ; \omega=2 ; t_{1}=0.3 \mathrm{~s} ;$ 


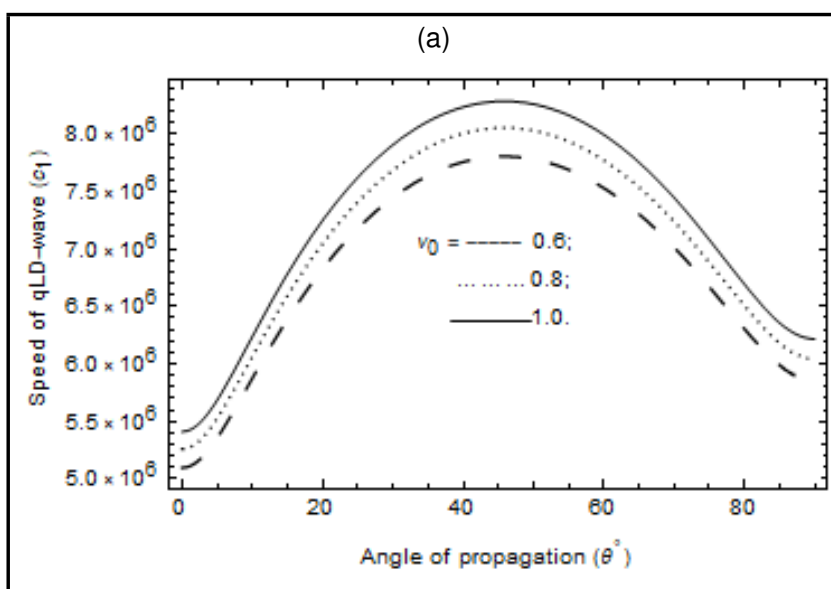

(b)

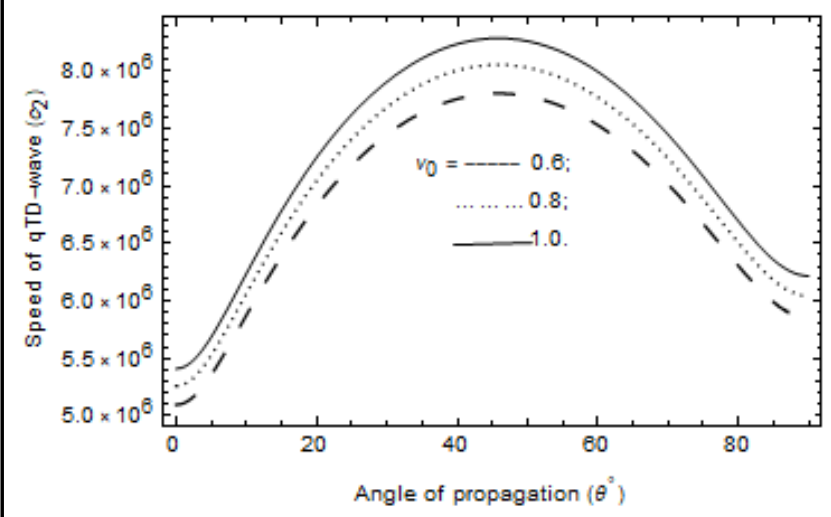

(c)

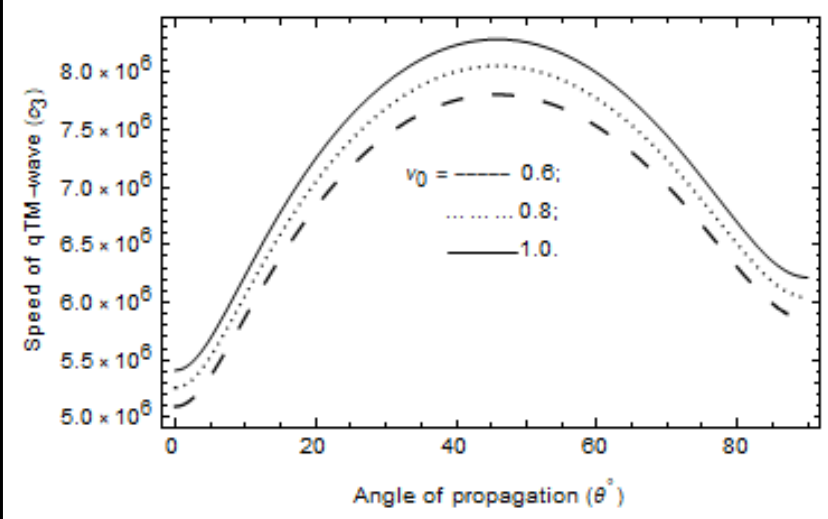

(d)

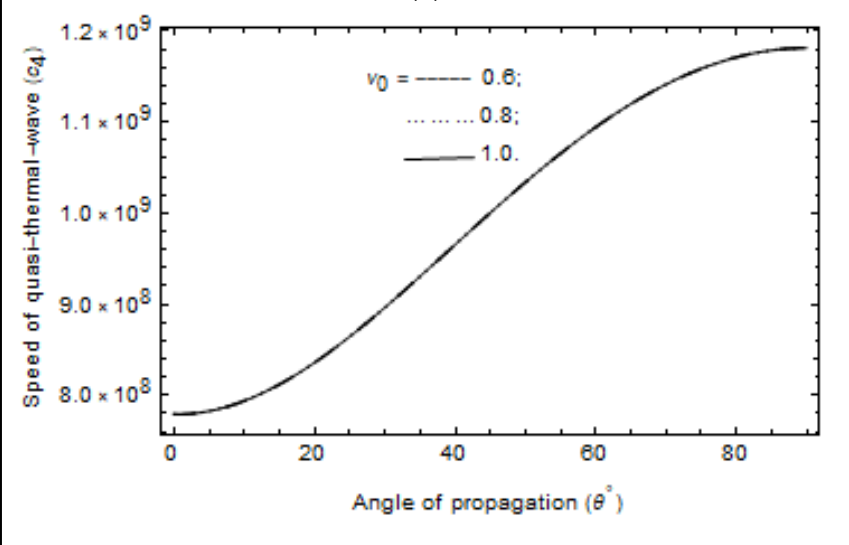

Figure 2. Variation of wave speeds $c_{i}, i=1,2,3$, and 4 versus angle of propagation for distinct values of thermal relaxation parameter $\nu_{0}$.

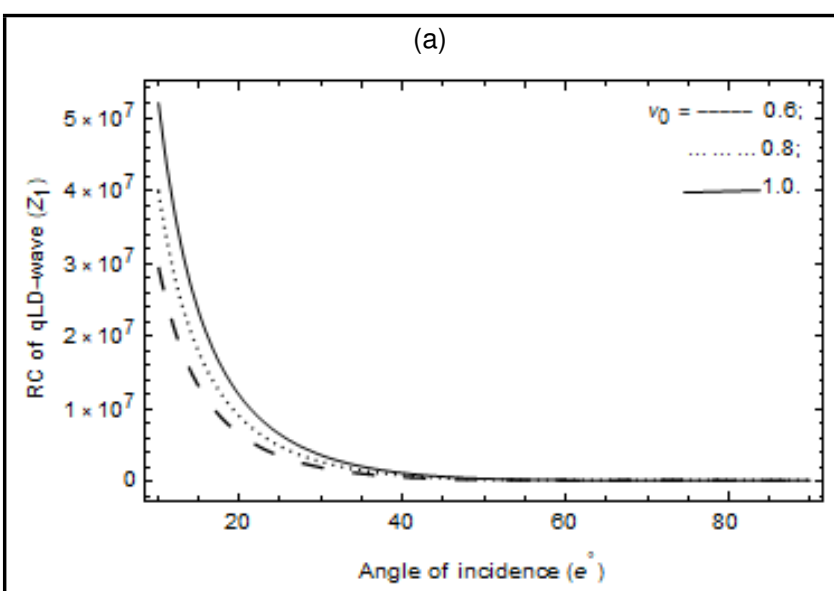

(b)

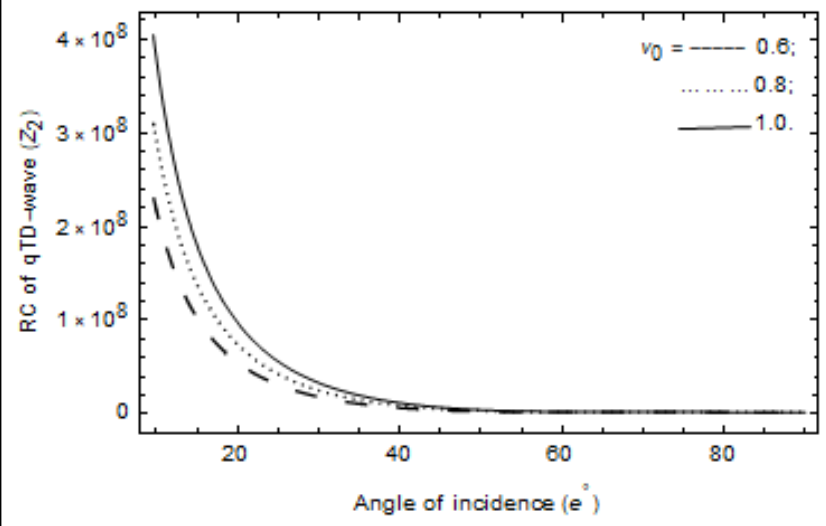

(c)

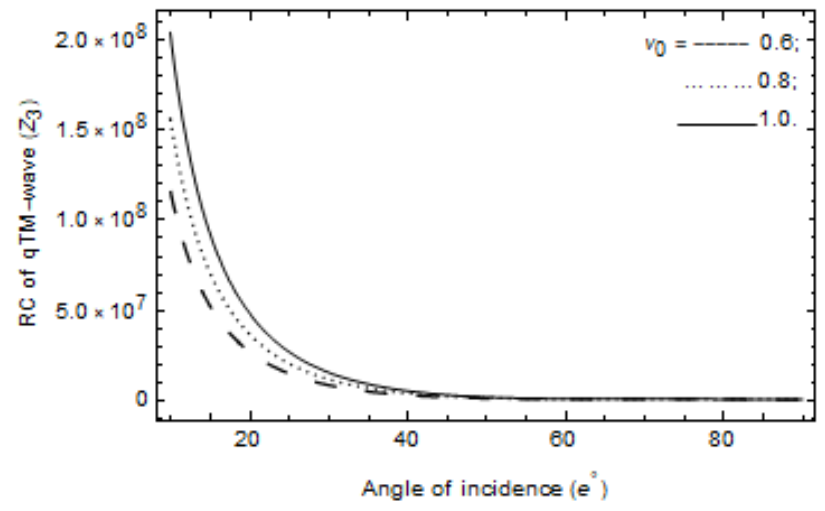

(d)

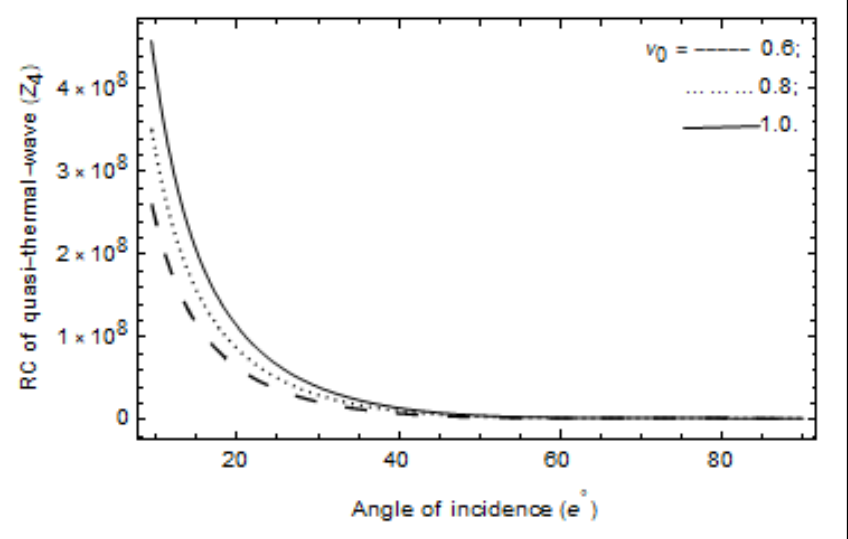

Figure 3. Variations of reflection coefficients $Z_{i}$ versus incidence angle for distinct values of thermal relaxation parameter $\nu_{0}$. 


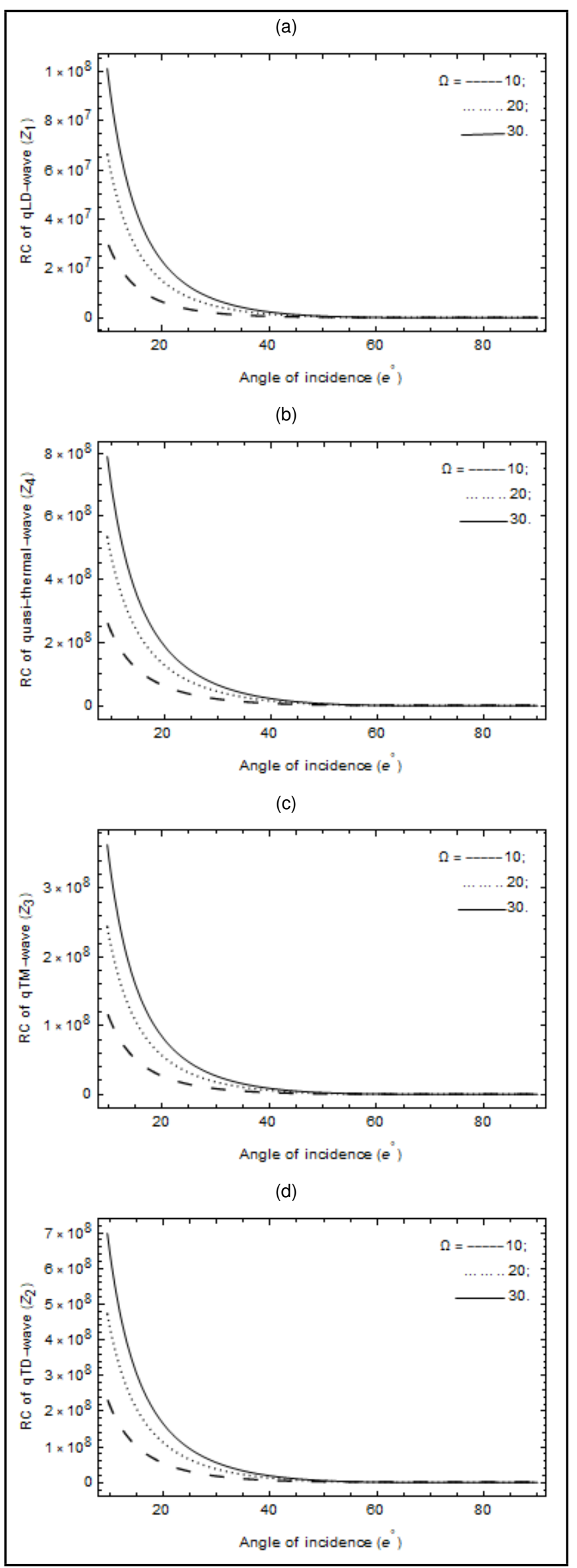

Figure 4. Variations of reflection coefficients $Z_{i}$ versus incidence angle for distinct values of $\Omega$.

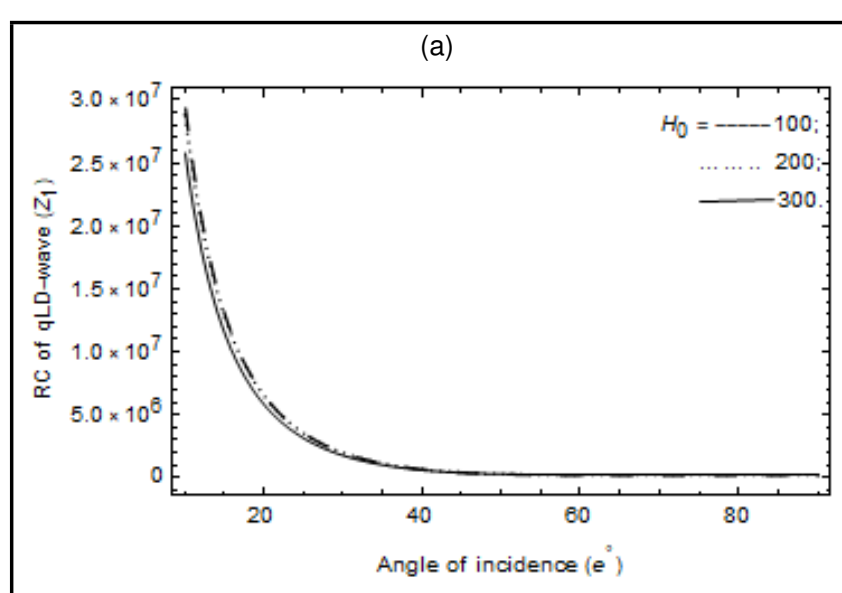

(b)

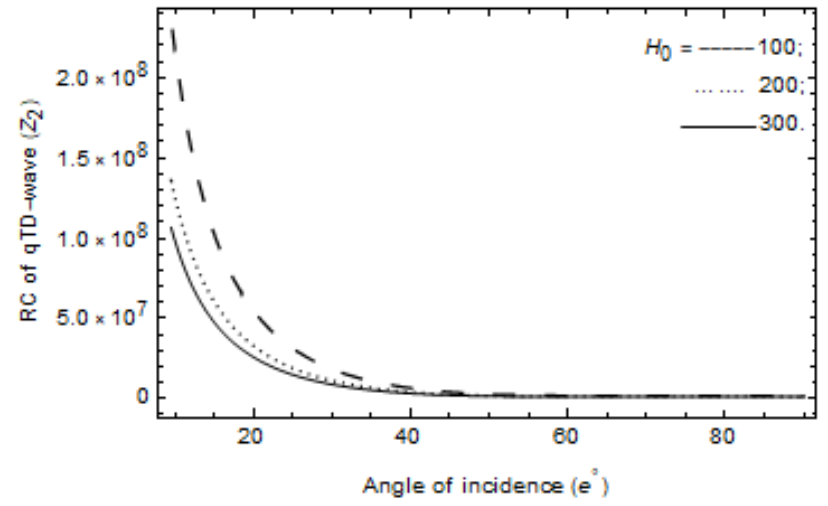

(c)

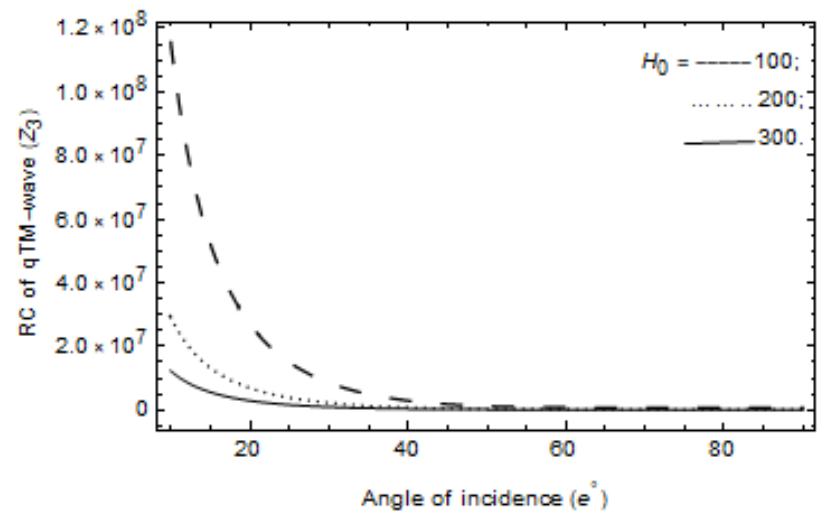

(d)

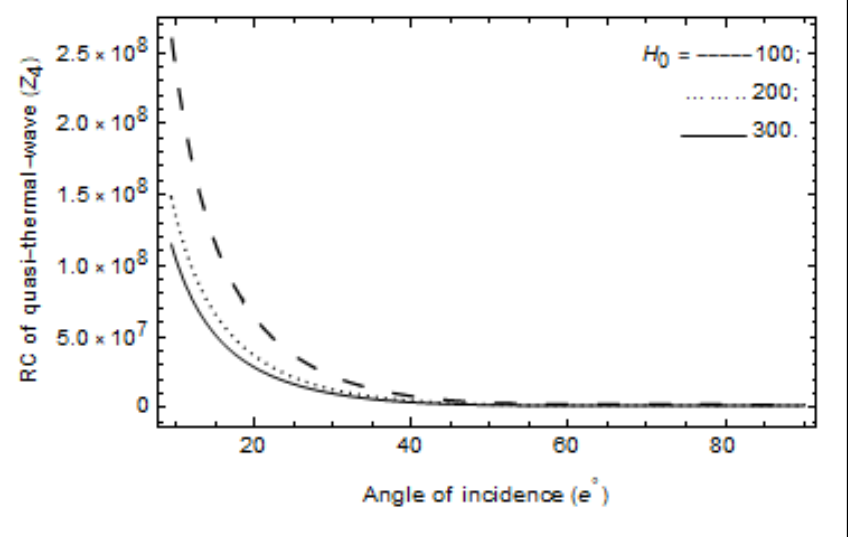

Figure 5. Variations of reflection coefficients $Z_{i}$ versus incidence angle for distinct values of magnetic field parameter $H_{0}$. 
$\nu_{0}=0.6 \mathrm{~s} ; C_{\nu}=0.787 \times 10^{3} \mathrm{Jkg}^{-1} \mathrm{deg}^{-1} ; T_{0}=2.93 \mathrm{~K} ;$ $K_{1}=0.0963 \times 10^{3} \mathrm{Jm}^{-1} \mathrm{~s}^{-1} \mathrm{deg}^{-1}$.

The angles of reflection and the wave numbers are computed using Snell's law. Also, the wave speeds are obtained from Eq. (20). Thus, the graphs are presented in Figs. 2-5; which show variations between the horizontal reflection coefficients ( $\mathrm{RC}$ or $Z_{i}$ ) when in particular, the $\mathrm{P}$-wave is incident with angle " $e$ ", for different values of thermal relaxation $\nu_{0}$, rotation $\Omega$ (angular velocity), and magnetic field parameters to ascertain the effects of these parameters on reflection coefficients of the waves under the G-L theory of thermo-elasticity.

Figure 2 illustrates the variation of qLD, qTD, qTM and quasi-thermal wave speeds $c_{1}, c_{2}, c_{3}$, and $c_{4}$ respectively against propagation angle for the distinct thermal relaxation parameter $\nu_{0}$. It is obvious from Fig. 2 that the speeds: $c_{1}, c_{2}$, and $c_{3}$ and of propagation or modulation of the wave at different points of propagation angle are dependent on the thermal constant $\nu_{0}$ of the medium. As $\nu_{0}$ increases, the speeds of the waves also increase; this is due to the thermal effects on the material. The speed of wave $c_{4}$ is unaffected by the thermal constant $\nu_{0}$. The maximum propagation of the speeds $c_{1}$, $c_{2}$, and $c_{3}$ of the waves occur near a propagation angle of $45^{\circ}$ while $c_{4}$ attains its maximum near a propagation angle of $90^{\circ}$. It is also observed that the speed of waves: $c_{1}, c_{2}$, and $c_{3}$ decreases after $45^{\circ}$ for an increasing angle of propagation.

Consequently, Fig. 3 depicts the variation of reflection coefficients $Z_{i}, i=1,2,3$ and 4 of qLD, qTD, qTM and quasithermal waves respectively against incidence angle for distinct values of thermal relaxation parameter $\nu_{0}$. It is obvious from Fig. 3 that the reflection coefficients $Z_{i}, i=1,2,3$ and 4 , attain their maxima at near normal incidence of propagation of the waves. When the thermal relaxation parameter $\nu_{0}$ increases, the reflection coefficients of the waves also increase owing to thermal effects on the material which tends to weaken its strength or its composition. Also, as the angle of incidence is increased the reflection coefficients uniformly decrease near $e^{\circ}>60^{\circ}$ to the grazing angle of incidence where it vanishes.

Figure 4(a-d) represents the effects of rotation on the reflection coefficients $Z_{i}$, of qLD, qTD, qTM and quasi-thermal waves respectively. Also this shows that the reflection coefficients have their maximum values close to the normal incidence of waves. Subsequently Fig. 4(a-d) depicts that the reflection coefficients increase when the rotation parameter increases. However, the reflection coefficients in Fig. 4(a-d) decrease uniformly from $e^{\circ}>40^{\circ}$ as they vanish at grazing angle of incidence. This means that the reflection coefficients of the waves are influenced by rotation effects and angle of incidence of the wave.

It is observed in Fig. 5(a-d) that the reflection coefficients, $Z_{i}(R C),(i=1,2,3,4)$ of $\mathrm{qLD}$ (P-wave), qTD (SV-wave), qTM (quasi-transverse micro-rotational wave) and quasi-thermal waves respectively, decrease for increasing angle of incidence. They also have decreasing behaviour with respect to increasing $H_{0}$. In Fig. $5, Z_{i}(R C),(i=1,2,3$, and 4 ), start with their curves at maximum values close to normal incidence and afterwards decrease in a uniform gradual manner when the angle of incidence approaches $40^{\circ}$ and finally vanish at grazing incidence. Furthermore, this is a physical explanation in that the higher the magnetic field, the more reflection of the wave becomes possible and its reflection coefficients possesses higher values in such a medium and the reverse is the case for decreased incident angle and magnetic fields. In Fig. 5(a), the refection coefficient shows a slight decrease with effects of the magnetic field unlike in Fig. 5(c) which is remarkably affected by the increased magnetic field.

\section{CONCLUSIONS}

The article investigated the propagation and reflection of magneto-thermoelastic plane waves at free surfaces of a rotating micropolar fibre-reinforced medium using Green and Lindsay thermo-elasticity theory. Four coupled reflected plane waves traveling in the medium; quasi-longitudinal displacement (qLD) waves, quasi-transverse displacement (qTD) waves, quasi-transverse micro-rotational (qTM) waves and quasi-thermal waves were observed. The characteristics of the propagation and reflection of the waves were made up of angular velocity of the medium $\Omega$, rotating micropolar fibrereinforced and thermal parameters under the influence of magnetic fields. It was deduced that these parameters have combined greatly to have remarkable degrees of influence on reflection coefficients, speeds and modulation of the waves from the numerically simulated graphs. This is such that the micropolar fibre-reinforcement parameters tend to decrease the modulation of the wave in a medium with impacts of magnetic fields, thermal effects and rotation. Generally, the propagation in the medium and the reflection coefficients are decreased for increased incident angles. It is noted that rotation produced an increasing behaviour in the reflection coefficients at certain values while magnetic field decreases the reflection coefficients in the modelled problem. Moreover, thermal parameters caused increasing behaviours in the reflection coefficients of qLD, (P-wave), qTD (SV-wave) and qTM (quasi-transverse micro-rotational wave) and quasi-thermal waves. Also it was deduced that reflection may not occur for certain incident angles in the medium especially near normal incidence and grazing incidence. It is noteworthy to state that the normal modes method used in this problem is helpful for achieving a successful investigation. Our modelled problem and results are similar to Sunita, Suresh and Kapil, ${ }^{30}$ if magnetic field and micropolar are neglected.

Moreover, the results from this article should prove useful for researchers in geomagnetic, structural designers, physics, the field of material sciences, optics, explorations such as oil and gas prospecting, new researchers in the field as well as experimental based study involving propagation and reflection of magneto-thermo-elastic plane waves at free surfaces of a rotating micropolar fibre-reinforced medium and also in mechanization fields of similar models such as earthquake analysis.

\section{ACKNOWLEDGEMENTS}

The World Academy of Sciences (TWAS) and COMSATS University Islamabad are appreciated for supporting and making the research works a success (FR \#: 3240293223). 


\section{REFERENCES}

${ }^{1}$ Lord, H. W. and Shulman, Y. A generalized dynamical theory of thermoelasticity, Journal of the Mechanics and Physics of Solids, 15, 299-309, (1967). https://dx.doi.org/10.1016/0022-5096(67)90024-5

${ }^{2}$ Green, A. E. and Lindsay, K. A. Thermoelasticity, Journal of Elasticity, 2, 1-7, (1972). https://dx.doi.org/10.1007/BF00045689

${ }^{3}$ Green, A. E. and Naghdi, P. M. Thermoelasticity without energy dissipation, Journal of Elasticity, 31, 189-208, (1993). https://dx.doi.org/10.1007/BF00044969

${ }^{4}$ Green, A. E. and Naghdi, P. M. On undamped heat waves in an elastic solid, Journal of Thermal Stresses, 15, 253-264, (1992). https://dx.doi.org/10.1080/01495739208946136

5 Green, A. E. and Naghdi, P. M. A re-examination of the basic postulates of thermomechanics, Proceedings of the Royal Society A, 432, 171-194, (1991). https://dx.doi.org/10.1098/rspa.1991.0012

${ }^{6}$ Eringen, A. C. Linear theory of micropolar elasticity, International Journal of Engineering Science, 5, 191-204, (1967). https://dx.doi.org/10.1016/0020-7225(67)90004-3

${ }^{7}$ McCarthy, M. F. and Eringen, A. C. Micropolar viscoelastic waves, International Journal of Engineering Science, 7, 447-458, (1969). https://dx.doi.org/10.1016/00207225(69)90032-9

${ }^{8}$ Kumar, R., Gogna, M. L., and Debnath, L. On Lamb's plane problem in micropolar viscoelastic halfspace with stretch, International Journal of Mathematics and Mathematical Sciences, 13 (2), 363-372, (1990). https://dx.doi.org/10.1155/S0161171290000540

${ }^{9}$ Lakes, R. S. and Benedict, R. L. Noncentrosymmetry in micropolar elasticity, International Journal of Engineering Science, 20 (10), 1161-1167, (1982). https://dx.doi.org/10.1016/0020-7225(82)90096-9

${ }^{10}$ Marin, M. Weak solutions in elasticity of dipolar porous materials, Mathematical Problems in Engineering, 2008, 1-8, (2008). https://dx.doi.org/10.1155/2008/158908

11 Marin, M. and Stan, G. Weak solutions in elasticity of dipolar bodies with stretch, Carpathian Journal of Mathematics, 29 (1), 33-40, (2013).

12 Biswas, P. K., Sengupta, P. R., and Debnath, L. Axisymmetric Lamb's problem in a semi-infinite micropolar viscolelastic medium, International Journal of Mathematics and Mathematical Sciences, 19, 815-820, (1996). https://dx.doi.org/10.1155/S0161171296001135

13 Singh, B. and Kumar, R. Reflection and refraction of plane waves at an interface between micropolar elastic solid and viscoelastic solid, International Journal of Engineering Science, 36, 119-135, (1998). https://dx.doi.org/10.1016/S0020-7225(97)00041-4
${ }^{14}$ Singh, B. and Kumar, R. Reflection and refraction of micropolar elastic waves at an interface between liquidsaturated porous solid and micropolar elastic solid, Proceedings of the National Academy of Sciences India, 70, 397-410, (2000).

15 Cowin, S. C. and Nunziato, J. W. Linear elastic materials with voids, Journal of Elasticity, 13, 125-147, (1983). https://dx.doi.org/10.1007/BF00041230

16 Nunziato, J. W. and Cowin, S. C. A nonlinear theory of elastic materials with voids, Archive for Rational Mechanics and Analysis, 72, 175-201, (1979). https://dx.doi.org/10.1007/BF00249363

${ }^{17}$ Puri, P. and Cowin, S. C. Plane waves in linear elastic materials with voids, Journal of Elasticity, 15, 167-183, (1985). https://dx.doi.org/10.1007/BF00041991

${ }^{18}$ Singh, B. Reflection of elastic waves from plane surface of a half-space with impedance boundary conditions, Geosciences Research, 2 (4), 242-253, (2017). https://dx.doi.org/10.22606/gr.2017.24004

19 Sengupta, P. R. and Nath, S. Surface waves in fibrereinforced anisotropic elastic media, Sadhana, 26, 363-370 (2001). https://dx.doi.org/10.1007/BF02703405

${ }^{20}$ Chattopadhyay, A., Venkateswarlu, R. L. K., and Saha, S. Reflection of quasi-P and quasi-SV waves at the free and rigid boundaries of a fibrereinforced medium, Sadhana, 27, 613-630, (2002). https://dx.doi.org/10.1007/BF02703354

${ }^{21}$ Chaudhary, S., Kaushik, V. P., and Tomar, S. K. Reflection/transmission of plane wave through a self-reinforced elastic layer between two half-spaces, Acta Geophysica Polonica, 52, 219-235, (2004).

22 Khan, A., Anya, I. A., and Kaneez, H. Gravitational effects on surface waves in non-homogeneous rotating fibrereinforced anisotropic elastic media with voids, International Journal of Applied Science and Engineering Research, 4 (5), 620-632, (2015).

23 Tauchert, T. R. Thermal stresses in micropolar elastic solids, Acta Mechanica, 11, 155-169, (1971). https://dx.doi.org/10.1007/BF01176553

${ }^{24}$ Chattopadhyay, A. and Choudhury, S. Propagation, reflection and transmission of magnetoelastic shear waves in a self-reinforced medium, International Journal of Engineering Science, 28, 485-495, (1990). https://dx.doi.org/10.1016/0020-7225(90)90051-J

${ }^{25}$ Kumar, R., Sharma, K. D., and Garg, S. K. Reflection of plane waves in transversely isotropic micropolar viscothermoelastic solid, Materials Physics and Mechanics, 22, 114, (2015).

26 Abd-Alla, A. M., Abo-Dahab, S. M., and Khan, A. Rotational effects on magneto-thermoelastic Stoneley, Love, and Rayleigh waves in fibre-reinforced anisotropic 
general viscoelastic media of higher order, Computers, Materials \& Continua, 53 (1), 49-72, (2017). https://dx.doi.org/10.3970/cmc.2017.053.052

27

Lata, P. Reflection and refraction of plane waves in a layered nonlocal elastic and anisotropic thermoelastic medium, Structural Engineering \& Mechanics, 66 (1), 113-124, (2018). https://dx.doi.org/10.12989/sem.2018.66.1.113

28 Schoenberg, M. and Censor, D. Elastic waves in rotating media, Quarterly of Applied Mathematics, 31, 115-125, (1973). https://dx.doi.org/10.1090/qam/99708

29

Sinha, M. and Bera, R. K. Eigenvalue approach to study the effect of rotation and relaxation time in generalised thermoelasticity, Computers \& Mathematics with Applications, 46, 783-792, (2003). https://dx.doi.org/10.1016/S08981221(03)90141-6

30 Deswal, S., Sheokand, S. K., and Kalkal, K. K. Reflection at the free surface of fiber-reinforced thermoelastic rotating medium with two-temperature and phase-lag, Applied Mathematical Modelling, 65, 106-119, (2019). https://dx.doi.org/10.1016/j.apm.2018.08.004

31 Roy, I., Acharya, D. P., and Acharya, S. Propagation and reflection of plane waves in a rotating magneto-elastic fibrereinforced semi space with surface stress, Mechanics and Mechanical Engineering, 21, 1043-1061, (2017).

32 Singh, B. and Singh, S. J. Reflection of plane waves at the free surface of a fibre-reinforced elastic half-space, Sadhana, 29 (3) 249-257, (2004). https://dx.doi.org/10.1007/BF02703774

33 Singh, S. S. and Lianngenga, R. Effect of micro-inertia in the propagation of waves in micropolar thermoelastic materials with voids, Applied Mathematical Modelling, 49, 487497, (2017). https://dx.doi.org/10.1016/j.apm.2017.05.008

${ }^{34}$ Lianngenga, R. and Singh, S. S. Effect of thermal and micro-inertia on the refraction of elastic waves in micropolar thermoelastic materials with voids, International Journal for Computational Methods in Engineering Science and Mechanics, 19 (4), 240-252, (2018). https://dx.doi.org/10.1080/15502287.2018.1500656

\section{APPENDIX}

$$
\begin{aligned}
& C_{1}=\left\{\rho \left(J k^{2} K_{1}\left(\rho+H_{0}^{2} \mu_{0}^{2}\right)\left(\rho+H_{0}^{2} \varepsilon_{0} \mu_{0}^{2}\right)+\right.\right. \\
& C_{\nu}\left(H_{0}^{4} \varepsilon_{0} \mu_{0}^{4}\left(-i J \rho \omega+2 k^{2} A_{4} D_{3} t_{1}\right)+\rho^{2}(-i J \rho \omega+\right. \\
& \left.\left(-2 J \rho \Omega^{2}+J k^{2} D_{1}+J k^{2} D_{2}+2 k^{2} A_{4} D_{3}\right) t_{1}\right)- \\
& \rho H_{0}^{2} \mu_{0}^{2}\left(i J \rho \omega+\left(J \rho \Omega^{2}-J k^{2} D_{2}-2 k^{2} A_{4} D_{3}\right) \tau_{0}+\right. \\
& \left.\left.\left.\left.\varepsilon_{0}\left(i J \rho \omega+\left(J \rho \Omega^{2}-J k^{2} D_{1}-2 k^{2} A_{4} D_{3}\right) \tau_{0}\right)\right)\right)\right)\right\} / \\
& \left\{-J k^{2} \rho^{2} C_{\nu}\left(\rho+H_{0}^{2} \mu_{0}^{2}\right)\left(\rho+H_{0}^{2} \varepsilon_{0} \mu_{0}^{2}\right) t_{1}\right\} ; \\
& C_{2}=\left\{\frac { 1 } { k ^ { 2 } } c ^ { 4 } \left(k ^ { 2 } \left(2 J K_{1} \rho^{3} \Omega^{2}-2 k^{2} K_{1} \rho^{2} A_{4} D_{3}+\right.\right.\right. \\
& i J \xi \rho^{2} \omega \cos [\theta]^{2} T_{0} \beta_{1}+i J \xi \rho^{2} \omega \sin [\theta]^{2} T_{0} \beta_{1}+ \\
& J K_{1} \rho^{2} \Omega^{2} H_{0}^{2} \mu_{0}^{2}-2 k^{2} K_{1} \rho A_{4} D_{3} H_{0}^{2} \mu_{0}^{2}+ \\
& i J \xi \rho \omega \cos [\theta]^{2} H_{0}^{2} T_{0} \beta_{1} \mu_{0}^{2}+J K_{1} \rho^{2} \Omega^{2} H_{0}^{2} \varepsilon_{0} \mu_{0}^{2}- \\
& \left.\left.\left.2 k^{2} K_{1} \rho A_{4} D_{3} H_{0}^{2} \varepsilon_{0} \mu_{0}^{2}\right)\right)\right\} / \\
& \left\{-J k^{2} \rho^{2} C_{\nu}\left(\rho+H_{0}^{2} \mu_{0}^{2}\right)\left(\rho+H_{0}^{2} \varepsilon_{0} \mu_{0}^{2}\right) t_{1}\right\} ; \\
& C_{3}=\left\{-\frac{1}{k^{4}}\left(k ^ { 4 } \rho \operatorname { s i n } ^ { 2 } [ 2 \theta ] A _ { 2 } ^ { 2 } \left(J k^{2} K_{1}+\right.\right.\right. \\
& \left.C_{\nu}\left(-i J \rho \omega+2 k^{2} A_{4} D_{3} t_{1}\right)\right)+ \\
& 4\left(J \rho \left(i \rho \omega C _ { \nu } \left(\rho \Omega^{2}\left(\rho\left(4 i^{2} \omega^{2}+\Omega^{2}\right)-k^{2} D_{2}\right)+\right.\right.\right. \\
& \left.D_{1}\left(-k^{2} \rho \Omega^{2}+k^{4} D_{2}\right)\right)+ \\
& k^{2}\left(-\rho \Omega^{2}\left(K_{1} \rho\left(4 i^{2} \omega^{2}+\Omega^{2}\right)+i \xi \omega T_{0} \beta_{1}\right)+\right. \\
& k^{2} D_{1}\left(K_{1} \rho \Omega^{2}-k^{2} K_{1} D_{2}+i \xi \omega \cos [\theta]^{2} T_{0} \beta_{1}\right)+ \\
& \left.\left.\left.\left.k^{2} D_{2}\left(K_{1} \rho \Omega^{2}+i \xi \omega \sin ^{2}[\theta] T_{0} \beta_{1}\right)\right)\right)\right)\right\} / \\
& \left\{-J k^{2} \rho^{2} C_{\nu}\left(\rho+H_{0}^{2} \mu_{0}^{2}\right)\left(\rho+H_{0}^{2} \varepsilon_{0} \mu_{0}^{2}\right) t_{1}\right\} ;
\end{aligned}
$$

$$
\begin{aligned}
C_{4} & =\left\{\frac { 1 } { k ^ { 4 } } A _ { 4 } \left(i ^ { 2 } k ^ { 2 } \operatorname { s i n } [ \theta ] A _ { 5 } \left(-2 i k^{2} K_{1} \rho \omega \Omega \cos [\theta]+\right.\right.\right. \\
& k^{2} K_{1} \rho \Omega^{2} \sin [\theta]+2 i^{2} \rho^{2} \omega^{2} \Omega \cos [\theta] C_{\nu}- \\
& i \rho^{2} \omega \Omega^{2} \sin [\theta] C_{\nu}-k^{2} \cos [\theta]^{2} \sin [\theta] A_{2}\left(k^{2} K_{1}-i \rho \omega C_{\nu}\right)- \\
& k^{2} \cos [\theta]^{2} \sin [\theta] A_{3}\left(k^{2} K_{1}-i \rho \omega C_{\nu}\right)-k^{4} K_{1} \sin [\theta] D_{1}+ \\
& \left.\left.\left.i k^{2} \rho \omega \sin [\theta] C_{\nu} D_{1}+i k^{2} \xi \omega \sin [\theta] T_{0} \beta_{1}\right)\right)\right\} \\
& \left\{-J k^{2} \rho^{2} C_{\nu}\left(\rho+H_{0}^{2} \mu_{0}^{2}\right)\left(\rho+H_{0}^{2} \varepsilon_{0} \mu_{0}^{2}\right) t_{1}\right\} .
\end{aligned}
$$

